For numbered affiliations see end of article.

Correspondence to: $\mathrm{H}$ Zhao hongzhao2005@aliyun.com Cite this as: BMJ 2022;376:e070533 http://dx.doi.org/10.1136/bmi-2022-070533 Published: 25 February 2022
ACUPUNCTURE: HOW TO IMPROVE THE EVIDENCE BASE \section{Increasing the usefulness of acupuncture guideline recommendations}

Yu-Qing Zhang and colleagues examine the progress and pitfalls in guideline recommendations for acupuncture and provide suggestions for improvement

Yu-Qing Zhang, $1,2,3,4$ Liming Lu, ${ }^{5}$ Nenggui Xu, ${ }^{5}$ Xiaorong Tang, ${ }^{5}$ Xiaoshuang Shi, ${ }^{3}$

Alonso Carrasco-Labra, ${ }^{6}$ Holger Schünemann, ${ }^{2}$ Yaolong Chen, ${ }^{7,8}$ Jun Xia, ${ }^{4,9}$ Guang Chen, ${ }^{10}$ Jianping Liu, ${ }^{11}$ Baoyan Liu, ${ }^{12}$ Jiyao Wang, ${ }^{13}$ Amir Qaseem, ${ }^{14}$ Xianghong Jing, ${ }^{3}$ Gordon Guyatt, ${ }^{2}, 15$ Hong Zhao ${ }^{3,16}$

http://dx.doi.org/10.13039/501100001809

National Natural Science Foundation of China

Acupuncture is the most widely used traditional and complementary medicine, used in 113 of 120 countries according to a 2019 World Health Organization report. ${ }^{3}$ In addition to registered acupuncturists, medical doctors, nurse practitioners, and chiropractors occasionally deliver acupuncture treatment. ${ }^{4}$ Despite its widespread use and considerable available evidence, until recently, clinical practice guidelines from conventional medical organisations rarely included recommendations on acupuncture. Clinical practice guidelines aim to optimise patient care, ${ }^{1}$ and must meet standards of trustworthiness to avoid misleading clinicians, patients, and other stakeholders. ${ }^{2}$ We begin by summarising the progress made then address deficiencies that limit the clinical usefulness of acupuncture guidelines, examine the barriers to inclusion of acupuncture in guidelines, and suggest how to overcome them.

\section{Which guidelines include acupuncture recommendations?}

More than 1300 consensus and evidence based clinical practice guidelines were published between
1991 and 2007, and these included 2189 recommendations on the use of acupuncture. ${ }^{5}$ Two thirds of these recommendations focused on pain related conditions. ${ }^{5}$ Guidelines that include acupuncture fall into three types: those including acupuncture with conventional interventions, those restricted to traditional and complementary medicine, and those covering acupuncture only.

Although researchers have criticised the exclusion of acupuncture interventions from clinical guidelines, ${ }^{5}$ conventional medicine is increasingly including acupuncture as one more relevant intervention. For example, between 2010 and 2020, 133 guidelines included more than 430 acupuncture recommendations worldwide (fig 1 )..$^{7}$ Over $70 \%$ of these guidelines were produced by conventional medical organisations, with the remainder published by organisations focused on traditional and complementary medicine in China, South Korea, and Australia. ${ }^{7}$ Acupuncture was most commonly recommended for musculoskeletal and connective tissue disease; neurological disorders; obstetrics, gynaecology, and women's health; oncology; and gastrointestinal disorders. ${ }^{-8}$ 


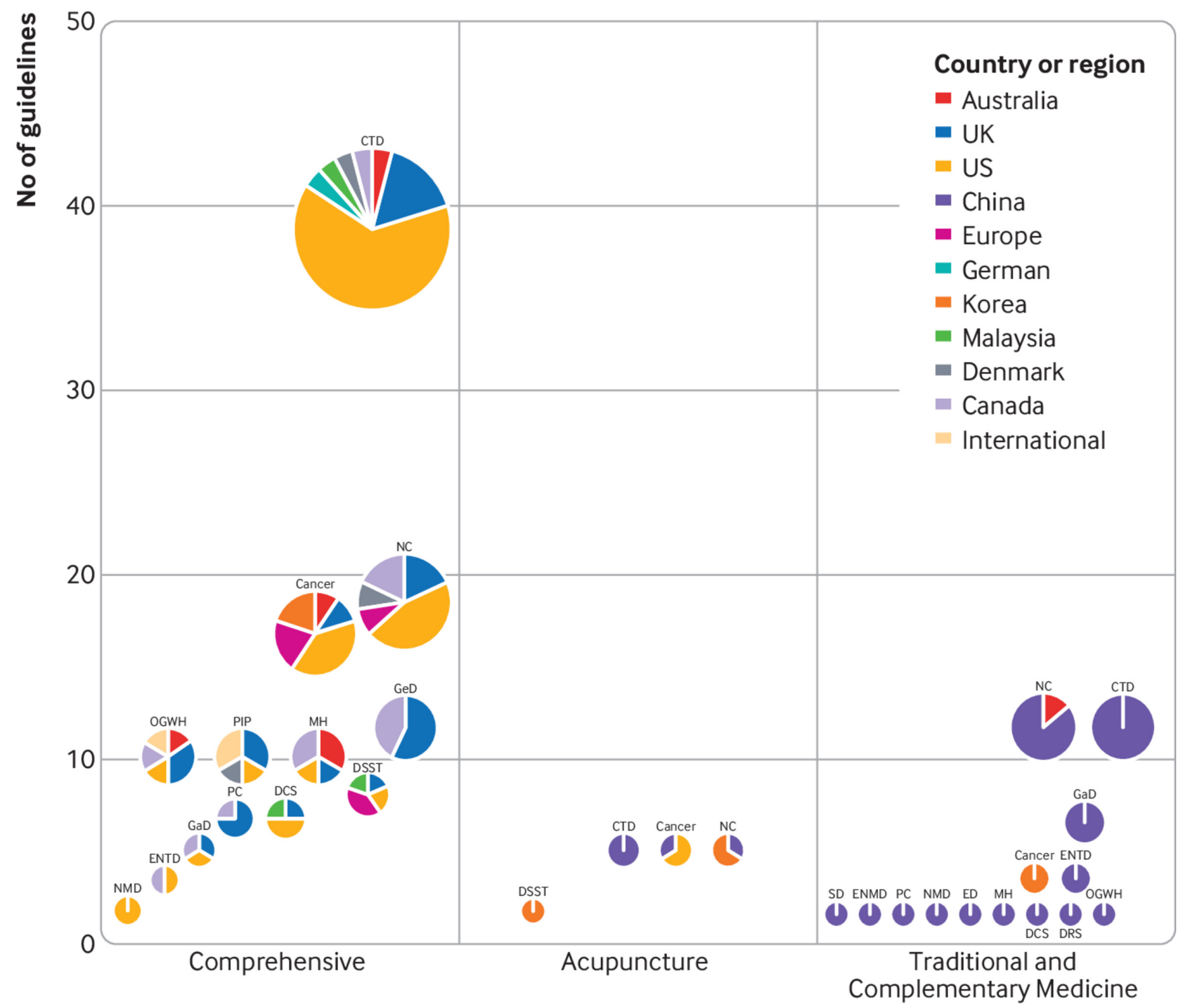

Type of guidelines

Fig 1 | Clinical guidelines that include acupuncture recommendations according to whether they are comprehensive (including conventional medicine), acupuncture only, or all traditional and complementary medicine

\section{Are acupuncture guidelines evidence based?}

Trustworthy guidelines are informed by systematic reviews of the best available evidence, including an assessment of the certainty of evidence and a rating of the strength of recommendations. ${ }^{1}$ Nearly half $(49 \%)$ of the guidelines that included acupuncture interventions relied on systematic reviews, which is a higher proportion than for guidelines in general medicine (31\% used systematic reviews). ${ }^{9}$ Roughly half of the guidelines used the GRADE approach to assessing evidence certainty and strength of recommendations, ${ }^{7}$ with other methods such as those of the Scottish Intercollegiate Guidelines Network, National Health and Medical Research Council, American College of Cardiology/American Heart Association, and Oxford Centre for Evidence-Based Medicine used in less than $5 \%$ of guidelines. $^{7}$

\section{How useful are current acupuncture guidelines?}

To be useful, clinical recommendations should explicitly describe the target patients with sufficient detail to allow clinicians to judge the extent of similarity to their patients. In acupuncture guidelines, as in some others, this is not always the case. ${ }^{10}$ Among acupuncture recommendations published in the past 10 years, less than $4 \%$ reported the severity of the underlying condition to which their recommendations apply, and less than $30 \%$ reported disease stage. 7 For example, in 2019, the China Association of Chinese Medicine published a guideline for the diagnosis and treatment of knee osteoarthritis using traditional Chinese medicine. ${ }^{11}$ It recommended using acupuncture for knee pain, swelling, and limited function without specifying the severity of the osteoarthritis. It is unclear to which patient population (mild, moderate, severe, or all) its recommendations apply. 
Failure to provide sufficient details regarding the interventions also limits clinicians' ability to implement the recommended acupuncture treatment. Just $40 \%$ of guidelines from conventional medicine organisations specified the types of acupuncture (eg, manual, electro-acupuncture, acupressure) they were recommending, and only $5 \%$ specified the range of points selected. ${ }^{7}$ The American College of Chest Physicians guideline on complementary therapies and integrative medicine for lung cancer suggested using acupuncture or related techniques as an adjunct treatment for nausea and vomiting from chemotherapy or radiation therapy (moderate certainty evidence, conditional

recommendation). ${ }^{12}$ However, the recommendation did not specify the types of acupuncture, the range of acupoints selected, or the frequency and duration of treatment.

When using recommendations, clinicians need to understand how a particular intervention compares with available alternatives. The net benefit of a specific intervention is likely to differ substantially depending on the comparator. Current acupuncture recommendations often fail to make the comparator explicit (box 1). ${ }^{7}$ Without a clear comparison, a recommendation is not actionable. ${ }^{13}$

Box 1: Examples of acupuncture recommendations that do and do not sufficiently define comparators

UK guideline on diagnosis and management of headaches in young people and adults ${ }^{14}$

The guideline from the National Institute for Health and Care Excellence concluded a net benefit of acupuncture over no treatment and recommended acupuncture. It also concluded that topiramate had net benefit over acupuncture and so recommended topiramate ${ }^{14}$ Korean medicine clinical practice guideline for lumbar herniated intervertebral disc in adults ${ }^{15}$

The recommendation favouring acupuncture for lumbar herniated intervertebral disc failed to specify whether the comparator is no intervention or other alternative intervention (eg, physical therapy) ${ }^{15}$

For clinicians to determine if the evidence justifies the recommendations, guidelines need to provide evidence on all "patient important" outcomes (benefits, harms, and burdens) for the competing interventions. Among guidelines that included acupuncture interventions in the past decade, $80 \%$ of the acupuncture specific guidelines reported outcomes to justify their recommendations. However, nearly half of the guidelines from conventional and complementary medicine do not report the outcomes evaluated. ${ }^{7}$ Moreover, failure to consider patients' values and preferences further limited the usefulness of acupuncture recommendations. A review of clinical guidelines including acupuncture found less than $20 \%$ explicitly considered patients' values and preferences (box 2). ${ }^{7}$

Box 2: Example of acupuncture recommendation not explicitly considering patients' values and preferences

Guidelines on recurrent urinary tract infection from the Canadian Society of Obstetricians and Gynaecologists ${ }^{16}$ suggest acupuncture as an alternative prevention strategy in women with recurrent infections who are unresponsive to or intolerant of antibiotic prophylaxis.

Continuous low dose antibiotics can cause antibiotic resistance, vaginal and oral candidiasis, and severe side effects such as skin rash and severe nausea. ${ }^{16} 17$ Patients who place a high value on avoiding antibiotic related adverse effects might prefer acupuncture prophylaxis regardless of their responsiveness to or tolerance of antibiotics.

If the panel had considered patient values it might have proposed acupuncture as an alternative to antibiotics in all women considering prophylaxis. A recommendation explicitly addressing patients' values would have a broader application beyond patients who are unresponsive to or intolerant of antibiotic prophylaxis.

\section{What barriers exist in acupuncture guideline implementation?}

Implementing an evidence based intervention into usual care takes an average of 17 years. ${ }^{18}$ Acupuncture is not an exception to this.

For example, less than a quarter of clinicians in the US and China follow evidence based guidelines on use of acupuncture to treat low back pain and assist in recovery from cerebral infarction. ${ }^{19-21}$ Barriers to implementation of acupuncture guidelines include personal factors related to physicians' knowledge and attitudes, guideline related factors, and external factors such as organisational constraints, lack of resources or time, workload, and facilitation. For instance, a recent UK study surveyed general practitioners' use of guidelines recommending acupuncture. ${ }^{22}$ Use of acupuncture was limited because of personal factors such as lack of awareness of the recommendations; external factors such as availability of practitioners to implement the intervention; and organisational constraints (clinical commissioning group controlling access).

Another study evaluated the implementation of evidence based traditional and complementary medicine guidelines on the care of inpatients after cerebral infarction in China. ${ }^{19}$ It found personal factors, including clinicians' disagreement with the recommendations and lack of training and knowledge in the use of guidelines; external factors related to resources (coverage, unavailability of acupuncturists, and patients' economic status); and social and clinical norms or culture were the most important constraints on uptake of acupuncture recommendations.

Cultural factors also affect policy making, the composition of guideline development committees, allocation of resources, and the acceptability of acupuncture. For example, when considering resource use, whether a country relies on universal coverage or insurance companies, whether patients can choose the healthcare providers, and whether healthcare providers have the choice of therapies can substantially affect whether acupuncture recommendations are followed.

A hospital focused study across 11 cities in China identified inconvenient usage (eg, failure to link recommendations to the hospital information system); guideline unawareness; practicality (eg, unclear, unfeasible, and impractical to implement); and lack of evidence from local context among the highest barriers.

Further research is needed in guideline implementation given the limited number of studies available.

\section{Do guidelines make recommendations for all questions of clinical interest?}

Patients and clinicians often encounter situations in which limited evidence is available to support the use of an intervention. Even when there is uncertainty, clinicians still prefer to have guidelines rather than relying on systematic reviews, as it saves time and effort. In a randomised controlled trial that presented 500 clinicians with a clinical scenario for which sparse evidence was available, $80 \%$ of participants preferred receiving a recommendation. ${ }^{2324}$ However, $22 \%$ of the guidelines from conventional medicine organisations that included evidence on acupuncture with low or very low certainty refrained from making recommendations. ${ }^{7}$ For example, the 2021 US Department of Veterans Affairs guidelines on primary care management of headache mentioned acupuncture but did not make any relevant recommendation. ${ }^{25}$ By contrast, the UK guidelines 
(rightly in our view) made a conditional recommendation suggesting up to 10 sessions of acupuncture over 5-8 weeks for prevention of chronic tension type headache despite low certainty of evidence compared with no treatment. ${ }^{14}$
A recent overview of reviews ${ }^{26}$ found low or very low certainty of evidence for moderate to large benefits from acupuncture in 50 diseases or conditions. Despite the substantial potential benefits documented, clinical guidelines disregarded acupuncture as an alternative in 15 of these diseases or conditions (table 1). 
Table 1 | Diseases and conditions for which acupuncture has a potential benefit (moderate or large effect) but is not included in clinical practice guidelines

\begin{tabular}{|c|c|c|c|c|c|}
\hline $\begin{array}{l}\text { Therapeutic area/disease or } \\
\text { condition }\end{array}$ & Comparators & Outcomes & Certainty of evidence & $\begin{array}{c}\text { Relative risk or standardised } \\
\text { mean differencet [Q to A } \\
\text { with } 95 \% \mathrm{Cl} \text { ? }\end{array}$ & Effect size ${ }^{\dagger}$ \\
\hline \multicolumn{6}{|l|}{ Connective tissue diseases } \\
\hline Fibromyalgia syndrome & Sham acupuncture & Sleep quality* & Low & $-0.46(-0.75$ to -0.18$)$ & Moderate \\
\hline \multicolumn{6}{|l|}{$\begin{array}{l}\text { Ear, nose, and throat } \\
\text { disorders }\end{array}$} \\
\hline Meniere's disease & Western medicine & Dizziness handicap inventory* & Very low & $-1.88(-3.06$ to -0.7$)$ & Large \\
\hline \multicolumn{6}{|l|}{ Eye disorders } \\
\hline Xerophthalmia & Western medicine & Symptom scores* & Very low & $-1.23(-2.08$ to -0.38$)$ & Large \\
\hline Xerophthalmia & Usual care & $\begin{array}{l}\text { Changes in Chronic Health } \\
\text { Evaluation II score }\end{array}$ & Low & 0.76 (0.5 to 1.03$)$ & Large \\
\hline \multicolumn{6}{|l|}{ Gastrointestinal disorders } \\
\hline Peptic ulcer & Western medicine & Recurrence rate* & Very low & 0.35 (0.14 to 0.84$)$ & Large \\
\hline \multicolumn{6}{|l|}{ Genitourinary disorders } \\
\hline Chronic kidney disease & Usual care & Depression* & Very low & $-0.72(-1.2$ to -0.24$)$ & Large \\
\hline Chronic kidney disease & Usual care & $\begin{array}{l}\text { Pittsburgh Sleep Quality } \\
\text { Index* }\end{array}$ & Very low & $-0.55(-0.96$ to -0.14$)$ & Moderate \\
\hline \multicolumn{6}{|l|}{ Mental health } \\
\hline Alcohol use disorder & Sham acupuncture & Alcohol craving* & Very low & $-1(-1.79$ to -0.21$)$ & Large \\
\hline Alcohol use disorder & No intervention & $\begin{array}{l}\text { Alcohol withdrawal } \\
\text { symptoms* }\end{array}$ & Low & $-0.54(-0.93$ to -0.14$)$ & Moderate \\
\hline Chronic fatigue syndrome & Sham acupuncture & Mental fatigue* & Very low & $-1.99(-3.67$ to -0.31$)$ & Large \\
\hline Opioid use disorder & No intervention & Craving for opioid* & Low & $-1.26(-1.73$ to -0.78$)$ & Large \\
\hline \multicolumn{6}{|l|}{ Neurological conditions } \\
\hline $\begin{array}{l}\text { Children with autism spectrum } \\
\text { disorder }\end{array}$ & $\begin{array}{l}\text { Behavioural and educational } \\
\text { interventions }\end{array}$ & $\begin{array}{l}\text { Overall Childhood Autism } \\
\text { Rating Scale* }\end{array}$ & Low & $-1.87(-3.21$ to -0.53$)$ & Moderate \\
\hline Carpal tunnel syndrome & Sham acupuncture & $\begin{array}{l}\text { Pain relief (at week } 4 \text { after } \\
\text { randomisation)* }\end{array}$ & Low & $-1.14(-1.98$ to -0.3$)$ & Large \\
\hline Carpal tunnel syndrome & Western medicine & $\begin{array}{l}\text { Pain relief (at week } 5 \text { after } \\
\text { randomisation)* }\end{array}$ & Very low & $-1.05(-1.64$ to -0.45$)$ & Large \\
\hline Tic disorder & Western medicine & Severity of tics* & Low & $-1.29(-1.87$ to -0.7$)$ & Large \\
\hline \multicolumn{6}{|c|}{ Obstetrics, gynaecology, and women's health } \\
\hline Menopausal syndrome & No intervention & $\begin{array}{l}\text { Hot flush frequency (>12 } \\
\text { weeks) }{ }^{*}\end{array}$ & Very low & $-0.7(-1.03$ to -0.36$)$ & Large \\
\hline Menopausal syndrome & No intervention & $\begin{array}{l}\text { Hot flush frequency («12 } \\
\text { weeks)* }\end{array}$ & Low & $-0.47(-0.78$ to -0.15$)$ & Moderate \\
\hline Menopausal syndrome & Western medicine & $\begin{array}{l}\text { Postmenopausal } \\
\text { osteoporosis-pain relief* }\end{array}$ & Very low & $-0.74(-1.08$ to -0.39$)$ & Large \\
\hline Menopausal syndrome & Sham acupuncture & $\begin{array}{l}\text { Quality of life (menopause } \\
\text { rating scale) at end of } \\
\text { treatment* }\end{array}$ & Very low & $-1.52(-1.77$ to -1.28$)$ & Large \\
\hline Menopausal syndrome & Sham acupuncture & $\begin{array}{l}\text { Quality of life (menopause } \\
\text { rating scale at follow-up 1-3 } \\
\text { months after treatment) }\end{array}$ & Very low & $-1.05(-1.28$ to -0.82$)$ & Large \\
\hline Postpartum lactating & Standard care & $\begin{array}{l}\text { Adequacy ratio of lactation } \\
\text { within } 24 \text { hours after delivery }\end{array}$ & Low & 2.11 (1.29 to 3.43$)$ & Large \\
\hline Postpartum lactating & Standard care & $\begin{array}{l}\text { Adequacy ratio of lactation } \\
\text { within } 72 \text { hours after delivery }\end{array}$ & Low & 2.37 (1.33 to 4.22$)$ & Large \\
\hline \multicolumn{6}{|l|}{ Periprocedural care } \\
\hline Postoperative pain & Sham acupuncture & $\begin{array}{l}\text { Intensity of postoperative pain } \\
\text { on movement or cough* }\end{array}$ & Very low & $-1.31(-1.71$ to -0.91$)$ & Large \\
\hline Postoperative pain & Sham acupuncture & $\begin{array}{l}\text { Postoperative resting pain } \\
\text { intensity at } 24 \mathrm{~h}^{\star}\end{array}$ & Very low & $-0.64(-0.89$ to -0.38$)$ & Moderate \\
\hline Postoperative pain & Sham acupuncture & $\begin{array}{l}\text { VAS scores } 24 \mathrm{~h} \text { after total } \\
\text { knee arthroplasty* }\end{array}$ & Very low & $-0.47(-0.87$ to -0.08$)$ & Moderate \\
\hline $\begin{array}{l}\text { Postoperative ileus after } \\
\text { abdominal surgery }\end{array}$ & Standard care & Time to first flatus* & Low & $-1.1(-1.54$ to -0.65$)$ & Large \\
\hline $\begin{array}{l}\text { Postoperative ileus after } \\
\text { abdominal surgery }\end{array}$ & Standard care & Time to first defecation* & Low & $-0.84(-1.29$ to -0.39$)$ & Large \\
\hline
\end{tabular}


Table 1 |Diseases and conditions for which acupuncture has a potential benefit (moderate or large effect) but is not included in clinical practice guidelines (Continued)

\begin{tabular}{|c|c|c|c|c|c|}
\hline $\begin{array}{l}\text { Therapeutic area/disease or } \\
\text { condition }\end{array}$ & Comparators & Outcomes & Certainty of evidence & $\begin{array}{l}\text { Relative risk or standardised } \\
\text { mean differencet [Q to } \mathrm{A} \\
\text { with } 95 \% \mathrm{Cl} \text { ? ] }\end{array}$ & Effect size $^{\dagger}$ \\
\hline $\begin{array}{l}\text { Postoperative ileus after } \\
\text { abdominal surgery }\end{array}$ & Standard care & $\begin{array}{l}\text { Time to bowel sound } \\
\text { recovery* }\end{array}$ & Low & $-1.2(-1.92$ to -0.47$)$ & Large \\
\hline
\end{tabular}

* Better outcome when the value is lower.

† For dichotomous outcomes, we chose relative risk (RR) of 2.0 or 0.5 as the reference for small and large effects, respectively ( $R R \geq 2.0$ or $R R \leq 0.5$ as large, $0.5<R R<2.0$ as small). For continuous outcomes, we used the standardised mean difference (SMD) of 0.4 and 0.7 as the reference for small, moderate, and large effects (SMD $<0.4$ as small, $0.4 \leq S M D<0.7$ as moderate, $\geq 0.7$ as large).

\section{Recommendations}

\section{Joint guideline development and definitive integration into patient care}

When deciding on treatment, patients should have access to evidence supporting all reasonable care options regardless of whether they belong to conventional, traditional, or complementary medicine. When existing guidelines have compared acupuncture with other interventions, acupuncture specific guidelines can provide more specificity by considering different forms of acupuncture.

Conventional medical societies should collaborate with specialised acupuncture medical associations to increase the consideration of acupuncture treatments in recommendations. Collaboration can occur at two levels-firstly, in establishing joint priorities on critical clinical questions to consider in clinical guidelines, including acupuncture. ${ }^{27}$ For instance, collaboration would facilitate comprehensive guidelines focusing on whether acupuncture for headache prevention should be recommended versus no intervention or usual care, while acupuncture specific guidelines can concentrate on the optimal type of acupuncture, point selection, and duration of acupuncture treatment. Secondly, collaboration could facilitate recruiting a broad range of clinical experts to guideline panels.

An example of such a type of collaboration is reflected in the production of the first global acupuncture guideline, a joint effort between conventional medicine, traditional and complementary medicine, and acupuncture specific societies. The World Federation of Acupuncture and Moxibustion Society recently developed the first international acupuncture guideline for treating depression. This effort included 21 expert panels from Australia, South Korea, Germany, Norway, the USA, and China. Several international medical societies have endorsed the guideline. Such joint efforts could improve efficiency, reduce research waste, facilitate guidelines dissemination, and encourage the uptake of recommendations in clinical practice.

\section{Produce more patient centric guidelines and recommendations}

When making acupuncture recommendations, guidelines should summarise all patient important benefits, harms, and burdens of the competing interventions and specify the values and preferences underlying their recommendations, including how they arrived at those values. To ensure a consistent understanding of outcomes when discussing evidence, guideline developers should create standard health outcome descriptors (or marker states) presentations in lay terms that describe the symptoms, time horizon (when and how long the outcome will persist), testing and treatment required, and consequences. ${ }^{28}$
Estimates of the relative importance patients place on different outcomes can come from systematic reviews of primary studies, surveys, or focus groups. Including patient partners in the panel may provide additional insights about patients' perspectives. ${ }^{29}$

\section{Increase use of existing extensive acupuncture evidence to inform recommendations}

Much evidence in many clinical areas, including acupuncture, is of low certainty. ${ }^{26}$ When guideline developers identify relevant clinical questions, they should use the best available evidence to formulate recommendations even if it is low certainty. Evidence matrices can help guideline developers to identify relevant evidence. A 2021 evidence mapping identified systematic reviews of 1402 randomised controlled trials in acupuncture for 77 diseases, across 12 therapeutic areas, including 138995 patients. ${ }^{26}$

\section{Improve reporting standards}

Acupuncture recommendations should improve reporting of patients' condition, disease state and severity, intervention, comparator(s), and outcomes. The International Standard for Reporting Items for Practice Guideline in Healthcare (RIGHT) includes an extension that covers reporting of acupuncture guidelines and recommendations.$^{3031}$ The Template for Intervention Description and Replication (TIDieR) and TIDieR-Placebo checklists help achieve optimal reporting of interventions and comparators. ${ }^{3233}$

\section{Conclusion}

Conventional medical communities increasingly consider acupuncture, contributing two thirds of all guidelines addressing acupuncture interventions. These recommendations are, however, still limited in their usefulness because of poor reporting of target populations, incomplete description of acupuncture intervention, failure to specify alternative care options, lack of comprehensive consideration of all patient important outcomes, failure to elucidate and apply patients' values and preferences, and disregard of a large portion of the available body of acupuncture evidence. While limited available evidence suggests that existing barriers to implementation of acupuncture recommendations have commonality across cultures, few research efforts have focused on implementing acupuncture guidelines.

Guideline developers should establish joint efforts to set research priorities between conventional and complementary medicine communities and follow the relevant reporting standards and checklists to produce clear, actionable, trustworthy, patient centred guidelines. A global approach to guideline implementation research is needed to increase the uptake and adherence of evidence based acupuncture guidelines in practice. 


\section{Key messages}

- Local, regional, or international medical organisations producing conventional guidelines, including western interventions, contributed two thirds of acupuncture related guidelines

- Failure to adequately characterise patient, intervention, and comparator undermines the usefulness of acupuncture recommendations

- Clinical practice guidelines often fail to consider acupuncture interventions despite evidence of benefit

- Establishing collaboration between conventional and traditional complementary medicine communities may help remedy the neglect of acupuncture

- Key necessary improvements in acupuncture guidelines include explicit consideration of patients' values and preferences, using entire body evidence in acupuncture, and a clearer description of interventions, comparators, and the patients to whom recommendations apply.

\section{AUTHOR AFFILIATIONS}

1 CEBIM (Center for Evidence Based Integrative Medicine)-Clarity Collaboration,

Guang'anmen Hospital, China Academy of Chinese Medical Sciences, Beijing, China

2

Department of Health Research Methods, Evidence, and Impact, McMaster University, Hamilton, Ontario, Canada

3 Institute of Acupuncture and Moxibustion, China Academy of Chinese Medical Sciences, Beijing, China

Nottingham Ningbo GRADE Centre, University of Nottingham Ningbo, China

South China Research Center for Acupuncture and Moxibustion, Medical College of Acu-Moxi and Rehabilitation, Guangzhou University of Chinese Medicine, Guangzhou, China

6 Center for Integrative Global Oral Health, School of Dental Medicine, University of Pennsylvania, Philadelphia, PA, USA

Institute of Health Data Science, Lanzhou University, China

WHO Collaborating Centre for Guideline Implementation and Knowledge Translation, Lanzhou, China

9

Division of Epidemiology and Public Health, School of Medicine, University of Nottingham, UK

Harvard Medical School, Harvard University, Boston, USA

Centre for Evidence-Based Chinese Medicine, Beijing University of Chinese Medicine, Beijing, China

12

China Academy of Chinese Medical Sciences, Beijing, China

13

Zhongshan Hospital, Fudan University, Shanghai, China

14

American College of Physicians, Philadelphia, Pennsylvania, USA

15 Department of Medicine, Faculty of Health Sciences, McMaster University, Hamilton, ON, Canada

16

Shenzhen Luohu District Hospital of TCM, Shenzhen, China

Contributors and sources: YQZ, HZ, LL, and NX conceived the study. XS and XT performed the data analysis. YQZ, LL, HZ, and XT wrote the manuscript's first draft. AC-L, HS, GG, YC, JX, GC, BL, JL, JW, $A Q$ and $X J$ reviewed and edited the manuscript. All authors interpreted the data, critically revised the manuscript for important intellectual content, and approved the final version. As co-first authors, YQZ, $\mathrm{LL}, \mathrm{NX}$, and XT contributed equally to this work. XJ and $\mathrm{HZ}$ contributed equally.
Competing interests: We have read and understood BMJ policy on declaration of interests and declare that the study was supported by the special project of "Lingnan Modernization of Traditional Chinese Medicine" within the 2019 Guangdong Provincial Research and Development Program (2020B1111100008); the Innovation Team and Talents Cultivation Program of the National Administration of Traditional Chinese Medicine (ZYYCXTD-C-202004); and the National Natural Science Foundation of China (82174527). The funders had no influence on study design, data collection, analysis, decision to publish, or manuscript preparation.

Provenance and peer review: Commissioned; externally peer reviewed.

This article is part of a collection funded by the special purpose funds for the belt and road, China Academy of Chinese Medical Sciences, National Natural Science Foundation of China, the National Center for Complementary and Integrative Health, the Innovation Team and Talents Cultivation Program of the National Administration of Traditional Chinese Medicine, the Special Project of "Lingnan Modernization of Traditional Chinese Medicine" of the 2019 Guangdong Key Research and Development Program, and the Project of First Class Universities and High-level Dual Discipline for Guangzhou University of Chinese Medicine. The BM/commissioned, peer reviewed, edited, and made the decision to publish. Kamran Abbasi was the lead editor for The BMJ. Yu-Qing Zhang advised on commissioning for the collection, designed the topic of the series, and coordinated the author teams. Gordon Guyatt provided valuable advice and guidance.

1 Institute of Medicine (US) Committee on Standards for Developing Trustworthy Clinical Practice Guidelines. Graham R, Mancher M, Miller Wolman D, et al. National Academies Press, 2011.

2 Eccles MP, Grimshaw JM, Shekelle P, Schünemann HJ, Woolf S. Developing clinical practice guidelines: target audiences, identifying topics for guidelines, guideline group composition and functioning and conflicts of interest. Implement Sci 2012;7:60. doi: 10.1186/1748-5908-7-60 pmid: 22762776

3 World Health Organization. WHO global report on traditional and complementary medicine. 2019. https://apps.who.int/iris/handle/10665/312342.

$4 \quad$ ljaz N, Boon H. Evaluating the international standards gap for the use of acupuncture needles by physiotherapists and chiropractors: a policy analysis. PLoS One 2019;14:e0226601. doi: 10.1371/journal.pone.0226601 pmid: 31846494

5 Dobos GJ, Kirschbaum B, Choi KE. The western model of integrative oncology: the contribution of Chinese medicine. Chin J Integr Med 2012;18:643-51. doi: 10.1007/s11655-012-1200-1 pmid: 22936317

6 Cho HW, Hwang EH, Lim B, etal. How current clinical practice guidelines for low back pain reflect traditional medicine in East Asian countries: a systematic review of clinical practice guidelines and systematic reviews. PLoS One 2014;9:e88027.

doi: 10.1371/journal.pone.0088027 pmid: 24505363

7 Tang $X$, Shi $X$, Zhao $H$, etal. Characteristics and quality of clinical practice guidelines addressing acupuncture interventions: a systematic survey of 133 guidelines and 433 acupuncture recommendations. BMJ Open (forthcoming).

8 Birch S, Lee MS, Alraek T, Kim TH. Overview of treatment guidelines and clinical practical guidelines that recommend the use of acupuncture: a bibliometric analysis. J Altern Complement Med 2018;24:752-69. doi: 10.1089/acm.2018.0092 pmid: 29912569

9 Lunny C, Ramasubbu C, Puil L, etal. Over half of clinical practice guidelines use non-systematic methods to inform recommendations: A methods study. PLoS One 2021;16:e250356. doi: 10.1371/journal.pone.0250356 pmid: 33886670

10 Brignardello-Petersen R, Carrasco-Labra A, Guyatt GH. How to interpret and use a clinical practice guideline or recommendation: users' guides to the medical literature. JAMA 2021;326:1516-23. doi: 10.1001/jama.2021.15319 pmid: 34665198

11 Working Group on Setting up Guidelines for Diagnosis and Treatment of Knee Osteoarthritis (Xibibing) in Orthopedics Section of China Association of Chinese Medicine. Clinical guidelines for diagnosis and treatment of knee osteoarthritis (Xibibing) in orthopedics and traumatology of traditional Chinese medicine. 2019;29:1-7.

12 Deng GE, Rausch SM, Jones LW, etal. Complementary therapies and integrative medicine in lung cancer: Diagnosis and management of lung cancer, 3rd ed: American College of Chest Physicians evidence-based clinical practice guidelines. Chest 2013;143(Suppl):e420S-36S. doi: 10.1378/chest.12-2364 pmid: 23649450

13 Neumann I, Santesso N, AkI EA, etal. A guide for health professionals to interpret and use recommendations in guidelines developed with the GRADE approach. J Clin Epidemiol 2016;72:45-55. doi: 10.1016/j.jclinepi.2015.11.017 pmid: 26772609

14 National Institute for Health and Care Excellence. Headaches: in over 12s: diagnosis and management. 2012. Updated Dec 2021. https://www.nice.org.uk/guidance/cg150.

15 Jun JH, Cha Y, Lee JA, etal. Korean medicine clinical practice guideline for lumbar herniated intervertebral disc in adults: an evidence based approach. Eur J Integr Med 2017;9:18-26.doi: 10.1016/j.eujim.2017.01.003

16 Epp A, Larochelle A. No. 250-recurrent urinary tract infection. J Obstet Gynaecol Can 2017;39:e422-31. doi: 10.1016/j.jogc.2017.08.017 pmid: 28935065

17 Langford BJ, Brown KA, Diong C, etal. The benefits and harms of antibiotic prophylaxis for urinary tract infection in older adults. Clin Infect Dis 2021;73:e782-91. doi: 10.1093/cid/ciab116 pmid: 33595621

18 Morris ZS, Wooding S, Grant J. The answer is 17 years, what is the question: understanding time lags in translational research. J R Soc Med 2011;104:510-20. doi: 10.1258/jrsm.2011.110180 pmid: 22179294 
19 Zhou L, Chen Y, Liu J, Liu Z, Gao Y. Evaluating the implementation of evidence-based TCM clinical practice guidelines for cerebral infarction. Eur I Integr Med 2014;6:147-55.doi: 10.1016/j.eujim.2014.03.006.

20 Ghildayal N, Johnson PJ, Evans RL, Kreitzer MJ. Complementary and alternative medicine use in the US adult low back pain population. Glob Adv Health Med 2016;5:69-78. doi: 10.7453/gahmi.2015.104 pmid: 26937316

21 Qaseem A, Wilt TJ, McLean RM, etalClinical Guidelines Committee of the American College of Physicians. Noninvasive treatments for acute, subacute, and chronic low back pain: a clinical practice guideline from the American College of Physicians. Ann Intern Med 2017;166:514-30. doi: 10.7326/M16-2367 pmid: 28192789

22 Czarnawska-lliev N. General practitioners' use of and attitudes to acupuncture in relation to the UK's National Institute for Health and Care Excellence (NICE) clinical guidelines-a pilot study. EurJ Integr Med 2016doi: 10.1016/j.eujim.2016.07.004

23 Neumann I, Alonso-Coello P, Vandvik PO, etal. Do clinicians want recommendations? A multicenter study comparing evidence summaries with and without GRADE recommendations. J Clin Epidemiol 2018;99:33-40. doi: 10.1016/j.jclinepi.2018.02.026 pmid: 29530644

24 Neumann I, Schünemann HJ. Guideline groups should make recommendations even if the evidence is considered insufficient. CMA/2020;192:E23-4. doi: 10.1503/cmaj.190144 pmid: 31932336

25 Veterans Affairs, Department of Defence. Clinical practice guide for the primary care management of headache.202.1 https://www.healthquality.va.gov/guidelines/Pain/headache/

26 Lu L, Zhang YQ, Ge S, etal. Evidence mapping and overview of systematic reviews of the effects of acupuncture therapies. BMJ Open (submitted).

27 Qaseem A, McLean RM, O'Gurek D, etalClinical Guidelines Committee of the American College of PhysiciansCommission on Health of the Public and Science of the American Academy of Family Physicians. Nonpharmacologic and pharmacologic management of acute pain from non-low back, musculoskeletal injuries in adults: a clinical guideline from the American College of Physicians and American Academy of Family Physicians. Ann Intern Med 2020;173:739-48. doi: 10.7326/M19-3602 pmid: 32805126

28 Wiercioch W, Nieuwlaat R, Akl EA, etal. Methodology for the American Society of Hematology VTE guidelines: current best practice, innovations, and experiences. Blood Adv 2020;4:2351-65. do: 10.1182/bloodadvances.2020001768 pmid: 32453843

29 Zhang Y, Coello PA, Guyatt GH, etal. GRADE guidelines: 20. Assessing the certainty of evidence in the importance of outcomes or values and preferences-inconsistency, imprecision, and other domains. J Clin Epidemiol 2019;111:83-93. doi: 10.1016/j.jclinepi.2018.05.011 pmid: 29800687

30 Chen Y, Yang K, Marušic A, etalRIGHT (Reporting Items for Practice Guidelines in Healthcare) Working Group. A reporting tool for practice guidelines in health care: the RIGHT statement. Ann Intern Med 2017;166:128-32. doi: 10.7326/M16-1565 pmid: 27893062

31 Tang C, Duan Y, Zhang Y, etalRIGHT (Reporting Items for Practice Guidelines in Healthcare) for Acupuncture Working Group. RIGHT for acupuncture: An extension of the RIGHT statement for clinical practice guidelines on acupuncture. J Clin Epidemiol 2021;139:330-9. doi: 10.1016/j.jclinepi.2021.05.021 pmid: 34091023

32 Hoffmann TC, Glasziou PP, Boutron I, etal. Better reporting of interventions: template for intervention description and replication (TIDieR) checklist and guide. BMJ 2014;348:91687. do: 10.1136/bmj.g1687 pmid: 24609605

33 Howick J, Webster RK, Rees IL, etal. TIDieR-Placebo: A guide and checklist for reporting placebo and sham controls. PLoS Med 2020;17:e1003294. doi: 10.1371/journal.pmed.1003294 pmid: 32956344

This is an Open Access article distributed in accordance with the Creative Commons Attribution Non Commercial (CC BY-NC 4.0) license, which permits others to distribute, remix, adapt, build upon this work non-commercially, and license their derivative works on different terms, provided the original work is properly cited and the use is non-commercial. See: http://creativecommons.org/licenses/by$\mathrm{nc} / 4.0 \%$ 